\title{
Health Promotion Practice
}

http://hpp.sagepub.com/

\section{Evaluation Framework for Translational Research: Case Study of Australia's Get Healthy Information and} Coaching Service ${ }^{\circledR}$

Blythe J. O'Hara, Adrian E. Bauman, Elizabeth G. Eakin, Lesley King, Marion Haas, Margaret Allman-Farinelli, Neville Owen, Magnolia Cardona-Morell, Louise Farrell, Andrew J. Milat and Philayrath Phongsavan

Health Promot Pract 2013 14: 380 originally published online 14 September 2012

DOI: $10.1177 / 1524839912456024$

The online version of this article can be found at:

http://hpp.sagepub.com/content/14/3/380

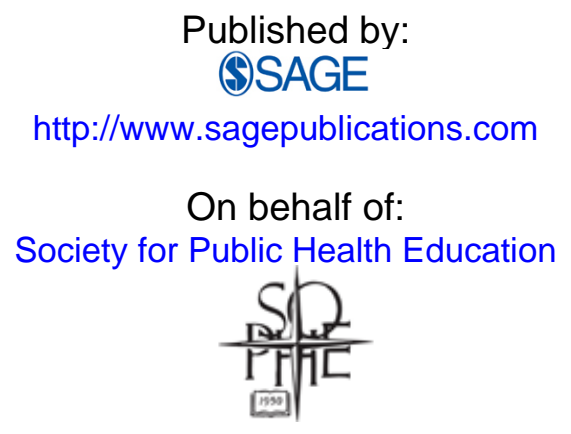

Additional services and information for Health Promotion Practice can be found at:

Email Alerts: http://hpp.sagepub.com/cgi/alerts

Subscriptions: http://hpp.sagepub.com/subscriptions

Reprints: http://www.sagepub.com/journalsReprints.nav

Permissions: http://www.sagepub.com/journalsPermissions.nav

>> Version of Record - May 2, 2013

OnlineFirst Version of Record - Sep 14, 2012

What is This? 


\title{
Evaluation Framework for Translational Research: Case Study of Australia's Get Healthy Information and Coaching Service ${ }^{\circledR}$
}

\author{
Blythe J. O'Hara, MAppSc ${ }^{1}$ \\ Adrian E. Bauman, $\mathrm{PhD}^{1}$ \\ Elizabeth G. Eakin, $\mathrm{PhD}^{2}$ \\ Lesley King, MPsych ${ }^{1}$ \\ Marion Haas, $\mathrm{PhD}^{3}$ \\ Margaret Allman-Farinelli, $\mathrm{PhD}^{1}$ \\ Neville Owen, $\mathrm{PhD}^{4}$ \\ Magnolia Cardona-Morell, $\mathrm{PhD}^{1}$ \\ Louise Farrell, $\mathrm{MPH}^{5}$ \\ Andrew J. Milat, MPH (Hons) ${ }^{5}$ \\ Philayrath Phongsavan, $\mathrm{PhD}^{1}$
}

The Get Healthy Information and Coaching Service ${ }^{\circledR}$ (GHS), a free government-funded telephone-delivered information and coaching service was launched in February 2009 by the Australian New South Wales state government. It represents the translation of research evidence applied in the real world ( $\mathrm{T} 4$ or Phase 4 translation), aimed at addressing the modifiable risk factors associated with the overweight and obesity. In controlled settings, it has been established that telephone-based lifestyle counseling programs are efficacious in reducing anthropometric and behavioral risk factors. This article presents the GHS case study as a population-wide intervention and describes the quasi-experimental evaluation framework used to evaluate both the process (statewide implementation) and impact (effectiveness) of the GHS in a real-world environment. It details the data collection, measures, and statistical analysis required in assessing the process of implementation-reach and recruitment, marketing and promotion, service satisfaction, intervention fidelity, and GHS setting up and operations costs-and in assessing the impact of GHS-increasing physical activity, improving dietary practices, and reducing

Health Promotion Practice

May 2013 Vol. 14, No. 3 380-389

DOI: $10.1177 / 1524839912456024$

(C) 2012 Society for Public Health Education body weight and waist circumference. The comprehensive evaluation framework designed for the GHS provides a method for building effectiveness evidence of a rare translation of efficacy trial evidence into populationwide practice.

Keywords: evaluation design; behavior change; obesity

\section{INTRODUCTION}

The prevalence of overweight and obesity continues to increase across the world, and Australia is no exception,

${ }^{1}$ University of Sydney, Sydney, New South Wales, Australia

${ }^{2}$ University of Queensland, Brisbane, Queensland, Australia

${ }^{3}$ University of Technology, Sydney, New South Wales, Australia

${ }^{4}$ Baker IDI, Heart and Diabetes Institute, Melbourne, Victoria, Australia

${ }^{5}$ NSW Ministry of Health, Sydney, New South Wales, Australia

Authors' Note: The NSW Ministry of Health provided funding to the University of Sydney to undertake an independent, comprehensive evaluation of the Get Healthy Service. No individual author received financial support. Please address correspondence to Blythe O'Hara, Prevention Research Collaboration, Sydney School of Public Health, Medical Foundation Building K25, University of Sydney, Sydney, New South Wales 2006, Australia; e-mail: blythe.ohara@sydney.edu.au. 
as nearly two thirds of the adult population are overweight or obese (Australian Bureau of Statistics, 2009), with associated direct and indirect costs in the order of $\$ 21$ billion (Colagiuri, Lee, et al., 2010). The causes are modifiable behavioral risk factors, such as increased consumption of energy-dense and nutritionally poor foods and inadequate levels of physical activity (Popkin \& Gordon-Larsen, 2004). To reverse the trends of inadequate levels of physical activity, poor nutrition, and increasing overweight and obesity, the population health importance of providing mass reach, evidencebased lifestyle improvement programs that are effective, relevant, accessible, and equitable is a public health priority.

The Australian Get Healthy Information and Coaching Service® (GHS), a free telephone-based information and coaching service, was launched in February 2009 to support individuals make lifestyle-based changes. As a government-funded service, GHS was introduced amid growing evidence for efficacious, mediated (i.e., not face-to-face) behavior-based interventions to address chronic disease risk behaviors such as physical inactivity, unhealthy eating, and overweight and obesity. The evidence base from systematic reviews has confirmed that telephone-based interventions (Eakin, Lawler, Vandelanotte, \& Owen, 2007; Goode, Reeves, \& Eakin, 2012) are effective in increasing physical activity, improving nutrition, and reducing weight in the short to medium term (3-6 months) across different populations, in a range of settings, and using different intervention modalities (Eakin et al., 2007).

However, published reports demonstrating the translation of this research into population-wide programs are limited. Whereas translational research from trials (Lindström et al., 2006) in other chronic disease domains such as diabetes is being championed in the primary health care setting for those with diabetes (Colagiuri, Vita, et al., 2010), lifestyle-based interventions and their effectiveness for addressing chronic disease risk factors and overweight and obesity in the general adult population remain largely untested and require further exploration, and the processes of translating research evidence to population-wide practice and policy need to be better understood (Goode et al., 2012; Jepson, Harris, Platt, \& Tannahill, 2010).

The importance of translational research is gaining prominence, with varying definitions of what translational research entails. One perspective describes translational research as technical linear processes (T1, basic scientific discovery; T2, evaluation of efficacy; T3, uptake and implementation into practice; and T4, focusing on evaluating the population-level health impact of interventions; Khoury, Gwinn, \& Ioannidis,
2010). Another considers this research area as iterative approaches that triangulate research, evidence, and policy and practice (Glasgow, Green, Taylor, \& Stange, 2012). A recent work on this area conceptualizes translational research as multilayered, staged models that emphasize not only the translation of research but also the importance of translating knowledge into evidencebased practice and vice versa (Rychetnik et al., 2012). Moreover, this perspective stresses researching and evaluating how and in what conditions evidence of intervention effectiveness in one setting can be translated to new settings and populations and to a larger scale. The goal is that a program has community-wide reach among the populations for whom it is intended. Regardless of the model, translation research articulates the significance of moving beyond the controlled settings afforded to traditional research trials to the real world and all the complexities it involves.

The GHS provides a rare example of a T4 translational research (Khoury et al., 2010; Rychetnik et al., 2012). Incumbent in the implementation of a program such as GHS is the responsibility to establish an evaluation framework that focuses on evaluating the replication and dissemination of efficacious trials and translating this knowledge into ongoing practice (Rychetnik et al., 2012). The characteristics of and the delivery of the GHS tailored to the needs of each individual, as described below, challenge the adequacy of the commonly used experimental evaluation designs, such as randomized controlled trials or group randomized controlled trials where communities, schools, or worksites can be randomly allocated. Other evaluation designs are often required for evaluating large-scale programs because of ethical or practical limitations preventing randomization. The tailored, self-directed, multicomponent elements of the GHS also preclude the intervention being delivered in a tightly controlled environment, with its impact likely to be influenced by external factors. In these situations, quasi-experimental or pretest and posttest evaluation designs may be all that is possible. This presents a constant tension between scientific rigor of evaluation and recognition of the delivery of a service that is highly context dependent.

Therefore, a comprehensive translatable evaluation framework that gives a balanced emphasis on assessing the implementation process and the effectiveness of GHS is required. The stages of research and evaluation model originally developed by Bauman and Nutbeam (2006) and further developed by Rychetnik et al. (2012) is used to guide portfolio development of evaluation substudies for the GHS. This model conceptualizes evaluation in several phases, from testing a new intervention in controlled conditions, evaluating efficacious 
programs under real-world conditions, and replicating effective programs to then disseminating and institutionalizing the program (Bauman \& Nutbeam, 2006). As evaluation progresses from controlled to real-world settings, so does the need to shift evaluation focus from assessing effectiveness to also monitoring the program delivery (implementation process). In this context, this article describes the evaluation framework used to assess the implementation and effectiveness of a real-world translational application of an efficacious telephone-based coaching service.

\section{METHOD}

\section{GHS Coverage Areas and Participants}

The GHS is offered to all residents living in the state of New South Wales, which has an estimated population of more than 7 million representing $34 \%$ of the population of Australia (Australian Bureau of Statistics, 2011). In 2010, the GHS was opened to residents living in the state of Tasmania and the Australian Capital Territory, which have a combined population of approximately 800,000 (Australian Bureau of Statistics, 2011).

The GHS is promoted to adults older than 18 years who are at risk of chronic disease because they do not meet healthy eating (National Health and Medical Research Council, 2003) or physical activity guidelines (Department of Health and Ageing, 2005) or who are overweight or obese. Most participants self-refer to the GHS and are recruited to contact the GHS (on a free-call phone number or website) through mass media marketing activities, local health service initiatives, and nongovernment organization promotions. Health professionals and general practitioners are also able to refer their clients to the GHS with an outbound GHS call made to these potential participants. A free interpreter service is available to participants from culturally and linguistically diverse backgrounds, as well as relevant telephonic services for participants who are deaf or who are hearing and speech impaired.

\section{Description of GHS}

The two service levels of GHS include an informationonly and a coaching service. The information-only service involves the delivery (via mail or e-mail) of an evidence-based information package that includes printed materials on healthy eating, physical activity, and achieving or maintaining a healthy weight, consistent with the Australian Guide to Healthy Eating (National Health and Medical Research Council, 2003) and National Physical Activity Guidelines (Department of Health and Ageing, 2005). In addition to the information package, a one-off information and advice session on these topics is available to callers at the time of the call. The website similarly replicates this information and provides detail on the GHS offerings (www.gethealthynsw.com.au).

The telephone coaching service includes 10 individually tailored calls provided by university-qualified health coaches. The coaching calls are based on behavior change/self-regulation principles designed to assist with goal setting, maintaining motivation, overcoming barriers, and making sustainable lifestyle changes (Palmer, Tibbs, \& Whybrow, 2003). Coaching calls are provided over a 6-month period on a tapered schedule, with a higher intensity of calls $(n=6)$ occurring in the first 12 weeks of the program to promote initiation of behavior change, and less frequent calls $(n=4)$ during the latter 14 weeks to promote maintenance and prevent relapse (Larimer, Palmer, \& Marlatt, 1999). Participants are able to cease coaching at any time during the 6-month program and are also able to reenroll in the program at the completion of the program.

Coaching participants undergo a medical screening via a telephone survey administered by the coach, where they are asked about any recent hospitalizations; chronic conditions; illnesses relating to the heart, lungs, or brain; physical conditions; pregnancy or breast feeding; mental health considerations; and special dietary considerations. If these questions highlight any relevant issue, the participant is referred to their general practitioner to obtain medical clearance before coaching can commence (Figure 1).

The GHS is provided by a private health insurance company under contract to the respective jurisdictional Governments and operates from 8 a.m. to 8 p.m. Monday to Friday. Health coaches are employed from a variety of health-related backgrounds, such as dietetics, exercise physiology, and psychology. The coaches undergo training and receive ongoing support in relation to behaviorally based health coaching and motivational interviewing (Rollnick \& Miller, 1995) and the GHS standardized protocol for asking the evaluation-related questions. Although the program is individualized with goal setting based on the lifestyle area (or areas) that the participant has identified as being important, participants are encouraged to set goals in relation to weight reduction or maintenance, physical activity, and healthy food choices.

\section{GHS Evaluation Design and Framework}

The primary goals of the evaluation framework are to assess the process of implementation and the 




FIGURE 1 Pathways for GHS Referral

SOURCE: Diagram courtesy of NSW Ministry of Health.

NOTE: GHS = Get Healthy Information and Coaching Service®.

impact of GHS (Bauman \& Nutbeam, 2006). This involves collecting information pertaining to GHS promotional activities and the delivery of the GHS (process evaluation) and participant outcomes (impact evaluation) using a pretest and posttest design to assess change in outcomes (Table 1). The framework reported here illustrates the present and ongoing components of the evaluation design, in addition to those components that have been completed, with the findings being used to inform the broader evaluation and refinement of GHS delivery. The University of Sydney Human Research Ethics Committee granted ethics approval for this research (reference numbers: 02-2009/11570, 20110906/14113, 03-2009/11614).

\section{Process Evaluation}

Data collection and measures. Process evaluation data are triangulated from a number of sources and collected by the GHS service provider, independent evaluators, and the Ministry of Health.

To assess reach and recruitment, GHS health coaches collect sociodemographic characteristics information from all participants, including gender, date of birth, residential postcode, highest level of education obtained, current employment status, language spoken at home, and Indigenous status. These questions are derived from the New South Wales Population Health Survey (Population Health Division, 2009). The postcode of participants is then used to determine SocioEconomic Indexes for Areas (Australia Bureau of Statistics, 2006) and Accessibility-Remoteness Index of Australia Plus (Australian Institute of Health and Welfare, 2004), which provide information regarding social disadvantaged and rurality. Furthermore, with regard to service type and level, information is collected by the GHS provider and the Ministry of Health 
TABLE 1

Description of Evaluation Component and Examples of Associated Data Collection

\begin{tabular}{|c|c|c|}
\hline Evaluation Component & Description & Examples of Data Collection \\
\hline \multirow[t]{13}{*}{ Process evaluation } & Reach and recruitment & $\begin{array}{l}\text { Number of calls and contacts to the GHS } \\
\text { Classification of calls and contacts to the GHS } \\
\text { GHS data collection on sociodemographic profile of participants } \\
\text { and their population representativeness }\end{array}$ \\
\hline & $\begin{array}{l}\text { Marketing and } \\
\text { promotion }\end{array}$ & $\begin{array}{l}\text { Type and extent of mass media advertising to encourage people } \\
\text { to contact GHS }\end{array}$ \\
\hline & & Campaign evaluation telephone survey \\
\hline & & $\begin{array}{l}\text { Type of local health service and non government organization } \\
\text { promotions }\end{array}$ \\
\hline & & Referral source collected by GHS \\
\hline & Service type and levels & GHS data collection on service usage \\
\hline & & $\begin{array}{l}\text { Type of service provided (information vs. coaching program and } \\
\text { conversion of service contacts to information and coaching) }\end{array}$ \\
\hline & $\begin{array}{l}\text { Service satisfaction and } \\
\text { fidelity }\end{array}$ & $\begin{array}{l}\text { Participant and service provider satisfaction and acceptability of } \\
\text { service }\end{array}$ \\
\hline & & Audit of coaching skills and processes \\
\hline & & Number of coaching calls delivered over what time period \\
\hline & & Predictors of coaching discontinuation/ completion \\
\hline & Economic appraisal & Service setup and delivery costs \\
\hline & & Promotional costs \\
\hline \multirow[t]{11}{*}{ Impact evaluation } & Anthropometric & Waist circumference (cm) \\
\hline & measures & Weight (kg) \\
\hline & & Height (cm) \\
\hline & $\begin{array}{l}\text { Behavioral risk factor } \\
\text { variables }\end{array}$ & $\begin{array}{l}\text { Nutrition (e.g., fruit and vegetable daily consumption, sweetened } \\
\text { drinks, takeaway meals, fat and fiber-related questions) }\end{array}$ \\
\hline & & $\begin{array}{l}\text { Physical activity (walking, moderate activity, vigorous activity } \\
\text { minutes and sessions) }\end{array}$ \\
\hline & Psychosocial variables & Psychological well-being \\
\hline & & Goal setting \\
\hline & & Stage of change \\
\hline & & Confidence \\
\hline & & Self-efficacy \\
\hline & & Social supports \\
\hline
\end{tabular}

NOTE: GHS = Get Healthy Information and Coaching Service®.

about the number and classification of calls and contacts to the service.

To assess the impact of marketing and promotion on GHS uptake, data on the extent, type, and timing of mass media advertising are collected. On behalf of the Ministry of Health, a random telephone survey of adults is carried out, by a market research agency, aimed at assessing mass media campaign reach, recall, awareness, understanding of campaign messages, behavioral intentions, and effectiveness of mass media advertising at reaching particular subgroups of the population. This survey is undertaken at baseline and includes tracking ongoing activity. A log of promotional activities undertaken by local health service and nongovernment organizations is also collected, and all GHS participants detail where they heard about GHS.

GHS satisfaction, fidelity, and acceptability among participants and the service provider are investigated, using qualitative methods, by the independent evaluators. To assess coaching fidelity, a review of a random 
sample of coaching calls is also undertaken to assess the coaching process and skills of the coach in meeting the aims of the program. This review includes questions in the domains of goal setting and behavior change, relationship development, process management and accountability, and the focus of the coaching sessions and is based on the coaching review frameworks developed by Moyers et al. (2007) and Lane et al. (2005). Factors associated with discontinuation/ completion are determined by examining the proportions of people who did not obtain a medical clearance, discontinued from the program prior to the 6-month time frame, and reenrolled in the program at the completion of the 6 months.

Economic appraisal of GHS is assessed through the collection of costs associated with the delivery and adoptions of the GHS at a government level. Associations between the cost of program implementation (e.g., cost of developing, promoting, and delivering the GHS) and effectiveness are measured in terms of the number of GHS users and their related behavior change outcomes. Individual-level expenditure data (Colagiuri, Vita, et al., 2010), such as direct health-related costs (visits to health professionals, hospitalization, medication use, etc.), direct nonhealth costs (gym subscription, exercise equipment, etc.), and indirect costs (e.g., sick days), are not considered in the economic evaluation.

\section{Impact Evaluation}

Data collection. Using a standard script and the validated measures (described in the Measures section), health coaches collect self-report data from all participants in the main GHS coaching cohort at baseline, 3 months, and 6 months as part of the minimum data set. A subsample of the GHS participants $(n=1,088)$ randomly drawn from the main GHS coaching cohort is also established by the independent academic evaluators. The sample size is based on the primary outcome of detecting mean reduction in body weight $(-2.5 \mathrm{~kg})$ from baseline to 12 months, with $80 \%$ power and $95 \%$ confidence interval, assuming .60 intraclass correlation for matched pair analysis and allowing $75 \%$ dropout at 12 months follow-up. This attrition rate is typical in community-based lifestyle change programs (Moroshko, Brennan, \& O'Brien, 2011).

The evaluators collect self-report data from the subsample of consenting participants at coaching entry and at 3 and 6 months (12 months from baseline) following the end of the coaching program (known as the "evaluation coaching subsample") by computer-assisted telephone interview (Figure 2). These data collection points allow for evaluation of anthropometric and behavioral risk factor changes from baseline to short term (3 months) and midterm (6 months) and for an assessment of maintenance of change 6 months after the end of formal coaching period (12-month data based on the evaluation coaching subsample).

Measures. For the main GHS coaching cohort sample, physical activity is assessed by three validated questions, which assess the number of times in a usual week participants engaged in the following: walking for 30 minutes or more, moderate-intensity physical activity for 30 minutes or more, and vigorous-intensity physical activity for 20 minutes or more (Smith, Marshall, \& Huang, 2005). In the evaluation coaching subsample, physical activity is assessed using the Active Australia Questionnaire and associated scoring protocol (Australian Institute of Health and Welfare, 2003), which is a six-item questionnaire asking participants about minutes of continuous walking for recreation, exercise or getting to and from places, and moderate- and vigorous-intensity physical activity, excluding household chores and gardening.

For nutrition-related behaviors, participants in the main GHS coaching cohort and the evaluation coaching subsample report the number of serves of fruit and vegetables consumed per day (Australia Bureau of Statistics, 1995), number of takeaway meals consumed per week, and the amount of sweetened drinks consumed per day (Population Health Division, 2009). Participants in the evaluation coaching subsample are asked an additional 20 questions derived from the Fat and Fibre Barometer (Wright \& Scott, 2000); the questionnaire asks about frequency of eating fruit and vegetables, reduced-fat cheeses, fried or roasted vegetables, red meat, processed meats, takeaway meals, crisps, biscuits, wholemeal pasta and rice, chocolates and lollies, legumes, and high-fiber biscuits; using cooking oils and fats; trimming fat off meat and poultry; and using low-fat milk and dairy products.

To examine psychosocial factors that may be associated with behavior change, the main GHS coaching cohort are asked questions pertaining to their stages of change (Prochaska, DiClemente, \& Norcoss, 1992) and confidence levels, and participants in the evaluation subsample are asked information regarding psychological well-being, goal setting, social support, and self-efficacy (Sallis, Grossman, Pinski, Patterson, \& Nader, 1987).

To minimize the potential biases and validate the self-reported (Han \& Lean, 1998) anthropometric measures and behavioral risk factors, a measurement validation study subsample was undertaken. This comprised a convenience sample $(n=38)$ with objectively measured 


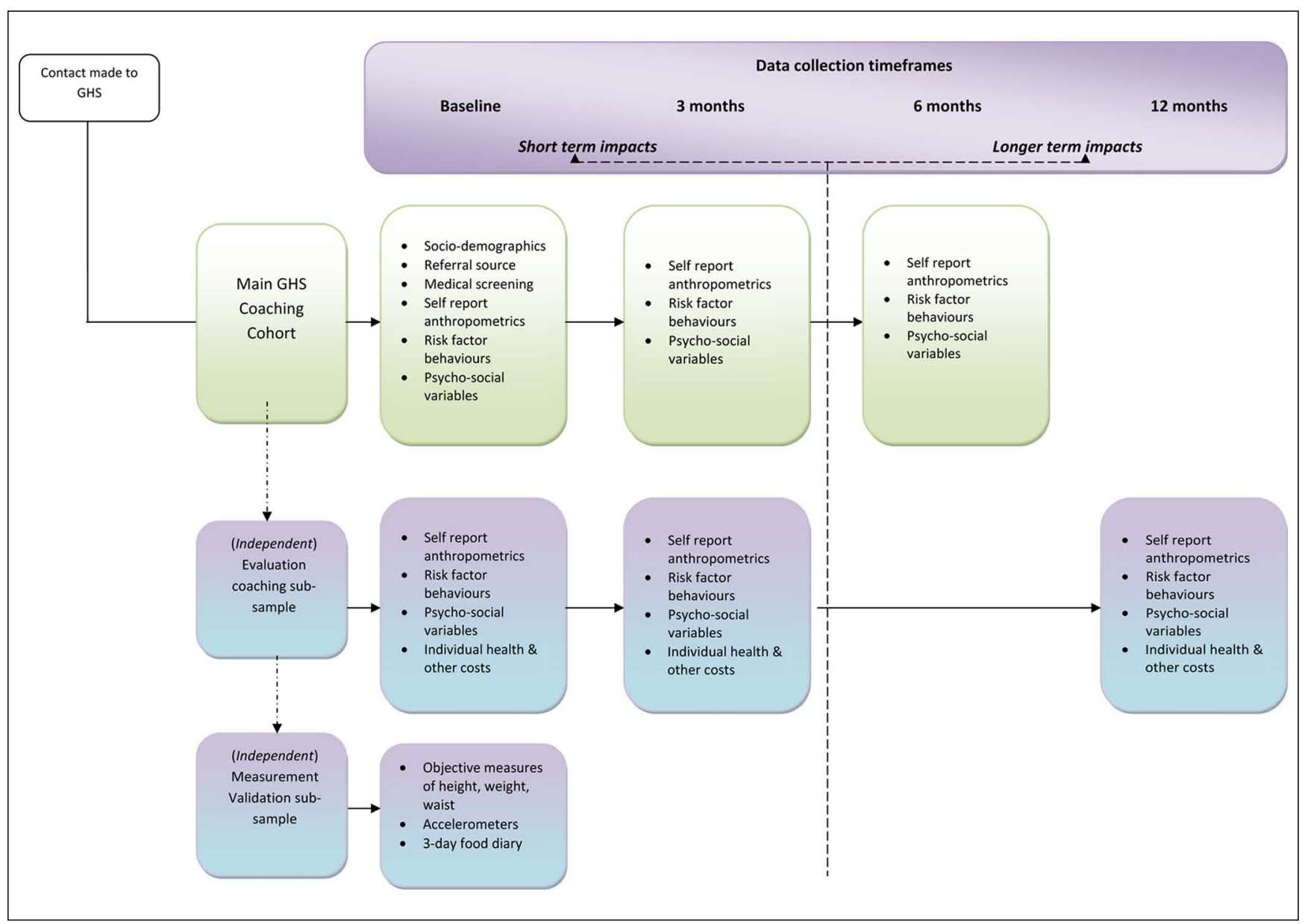

FIGURE 2 Overview of GHS Coaching Cohorts, Measurement Validation Subsample, Measures, and Data Collection Points NOTE: GHS = Get Healthy Information and Coaching Service®.

weight $(\mathrm{kg})$, height $(\mathrm{cm})$ and waist circumference $(\mathrm{cm})$ collected by researchers trained in conducting anthropometric measurements; and they collected 7-day accelerometer data to compare with self-reported physical activity measures and 3-day food diaries to validate self-report dietary behaviors (Caterson, 2011).

\section{Statistical Analyses}

Descriptive data related to the sociodemographic characteristics of GHS participants will be presented in counts and proportions. To test for statistical significance in relationships between variables, chi-square analyses will be conducted. To specifically investigate changes in anthropometrics (body weight, waist circumference) and risk factor behaviors (physical activity and nutrition) over time, multivariate modeling based on repeated measures generalized linear models will be estimated separately for each of the primary outcomes. This procedure enables assessment of individual trajectories over time when outcomes are measured at multiple points.

\section{DISCUSSION}

The GHS represents a unique case study of the translation of efficacy trial evidence into population-wide practice and an opportunity for researching and evaluating its effectiveness in the real-world environment. The existence of a comprehensive evaluation framework afforded to GHS signifies a rare example of investment in T4 research (Kessler \& Glasgow, 2011), acknowledging the important role translational research has in implementation science. Using the translational 
research model provided by Bauman and Nutbeam (2006), the GHS evaluation framework focuses on determining whether the GHS is an effective populationwide, public health program. The design of an evaluation framework that determines both implementation and effectiveness is critical for service delivery planners and the transfer of knowledge to evidence-based practice (Catford, 2009; Glasgow, Lichtenstein, \& Marcus, 2003; Goode et al., 2012; Milat, King, Baumam, \& Redman, 2012; Rychetnik et al., 2012). To do so within the complexities afforded by its real-world setting and using a range of criteria to judge the program's success is paramount (Rychetnik et al., 2012; Rychetnik, Frommer, Hawe, \& Shiell, 2002). Accordingly, the GHS evaluation uses a number of interrelated studies and a combination of quantitative and qualitative evaluation methods.

The process evaluation will provide critical implementation data particularly with regard to reach and recruitment, marketing and promotion, GHS satisfaction, fidelity, and acceptability and costs. Already the process evaluation has provided valuable information in terms of service reach and relevance (O'Hara, Phongsavan, Venugopal, \& Bauman, 2011). Analysis of the GHS users in the first 18 months of operations shows that the service is being used by populations living in socially and geographically disadvantaged regions and by those who have a higher chronic disease risk profile as measured by body mass index and waist circumference (O’Hara, Phongsavan, et al., 2011).

This evaluation has also identified some challenges with regard to the reach of the GHS, including underutilization by those who are non-English speaking (despite availability of translator services) and a lower proportion of male users compared with women (19.9\% vs. $80.1 \%$ )-male users were more likely to request the information pack only and not proceed to register in the coaching program. The service is now exploring ways to increase the appeal of GHS to men, including identifying referral and promotional pathways to GHS for this population group. The service has also identified usage by individuals from Aboriginal communities. However, to assist in closing the gap between mainstream and Aboriginal communities' health outcomes, there is a need to encourage even greater representation of those from Aboriginal communities.

The process evaluation has not only contributed to other continuous improvements in the service but also revealed important implementation challenges. Specifically, the importance of marketing and promotion in increasing population awareness of the GHS (O'Hara, Bauman, King, \& Phongsavan, 2011) through paid television advertising in the first year of the initiative indicates a clear dose-response relationship between intensive periods of GHS advertising and calls to the service. As a new service, ongoing and dedicated GHS marketing will be critical for raising awareness, ensuring growth in service use, and, in turn, increasing access among the individuals who would benefit from the service.

The impact evaluation will provide crucial information regarding the short- and long-term effectiveness of the GHS on participants; in particular, it will assess whether the 6-month coaching program has been effective in supporting individuals to lose weight, increase physical activity, and/or improve healthy eating habits. The longer term follow-up will assess the sustainability and maintenance of behavioral risk factor improvements. Similarly, determining the psychosocial and other factors that contribute to the effects of coaching intervention will result in improved implementation (i.e., recruitment and retention) of the GHS and contribute to the design of other similar interventions.

With regard to the scientific robustness of the evaluation framework, conducting an independent evaluation study and a measurement validation substudy to assess the self-report measures used redresses possible concerns raised about the social desirability associated with self-report data. Furthermore, the assessment of the validity of the self-report measures with objective data from measured anthropometry, 3-day food diary, and accelerometers has indicated acceptable agreement levels for population-wide evaluation studies (Caterson, 2011). Ideally, this measurement validation study would have also included a follow-up sample; however, pragmatically the time required to recruit participants within a certain geographical location to facilitate objective measurements rendered this follow-up impractical.

With the ongoing interpretation of GHS impact evaluation findings, some key methodological issues need to be acknowledged. The absence of a control or comparison group from the evaluation design makes it difficult to attribute with complete confidence any behavioral changes to the GHS, and the possibility of effects due to secular trends could not be dismissed. A pretest and posttest evaluation design has been chosen as a control community was not possible because GHS is offered to the entire state. Furthermore, the generalizability of the results may potentially be limited by loss to follow-up of participants withdrawing from the program. Intensive follow-up efforts are underway with participants in the independent evaluation cohort who had withdrawn from coaching to explore their reasons for withdrawal and obtain their behavioral risk factor and anthropometric profile; this may still yield some insights on those opting out from coaching prior to the completion of the 6 months. 


\section{- CONCLUSIONS}

The method of assessing the effectiveness of the GHS as a translational research example, by necessity, involves striking a balance between applying good, evidence-based practice and acceptable evaluation design that has to function in real-world, complex environments. Given the multiple components of the GHS that permit a degree of flexibility and variability in the intensity of coaching engagement, a multipronged evaluation strategy that triangulates multiple sources of data and studies and uses quantitative and qualitative evaluation methods is considered as the most appropriate methodology to assess the process of implementation and impact of the GHS. As a population-wide program developed from evidence-based, efficacious interventions, understanding the contextual factors that impede or facilitate program uptake and its effectiveness in a real-world environment underpins much of the GHS evaluation questions. As such, a comprehensive implementation evaluation framework is put in place to rigorously and systematically examine these underlying factors and will provide insights for other practitioners seeking to implement and evaluate large-scale programs for preventing chronic disease.

\section{REFERENCES}

Australia Bureau of Statistics. (1995). National nutrition survey. Canberra: Author.

Australia Bureau of Statistics. (2006). Information paper: An introduction to socio-economic indexes for areas (SEIFA). Canberra: Author.

Australian Bureau of Statistics. (2009). National health survey: Summary of results 2007-08. Canberra: Author.

Australian Bureau of Statistics. (2011). Australian demographic statistics. Canberra: Author.

Australian Institute of Health and Welfare. (2003). The Active Australia Survey: A guide and manual for implementation, analysis and reporting. Canberra: Author.

Australian Institute of Health and Welfare. (2004). Rural, regional and remote health: A guide to remoteness classifications. Canberra: Author.

Bauman, A. E., \& Nutbeam, D. (2006). Evaluation in a nutshell: A practical guide to the evaluation of health promotion programs. Sydney, New South Wales, Australia: McGraw-Hill.

Caterson, H. (2011). The validity of several physical activity, nutrition and overweight/obesity self report measurement instruments (Unpublished master's thesis). University of Sydney, Sydney, New South Wales, Australia.

Catford, J. (2009). Advancing the "science of delivery" of health promotion: Not just the "science of discovery." Health Promotion International, 24, 1-5.

Colagiuri, S., Lee, C. M. J., Colagiuri, R., Magliano, D., Shaw, J. E., Zimmet, P. Z., \& Caterson, I. D. (2010). The cost of overweight and obesity in Australia. Medical Journal of Australia, 192, 260-264.
Colagiuri, S., Vita, P., Cardona-Morrell, M., Singh, M. F., Farrell, L., Milat, A., . . Bauman, A. (2010). The Sydney Diabetes Prevention Program: A community-based translational study. BMC Public Health, 10, 328.

Department of Health and Ageing. (2005). National physical activity guidelines for adults. Canberra: Australian Government.

Eakin, E. G., Lawler, S. P., Vandelanotte, C., \& Owen, N. (2007). Telephone interventions for physical activity and dietary behaviour change. American Journal of Preventive Medicine, 32, 419-434.

Glasgow, R. E., Green, L. W., Taylor, M. V., \& Stange, K. C. (2012). An evidence integration triangle for aligning science with policy and practice. American Journal of Preventive Medicine, 42, 646-654.

Glasgow, R. E., Lichtenstein, E., \& Marcus, A. C. (2003). Why don't we see more translation of health promotion research to practice? Rethinking the efficacy-to-effectiveness transition. American Journal of Public Health, 93, 1261-1267.

Goode, A. D., Reeves, M. M., \& Eakin, E. G. (2012). Telephonedelivered interventions for physical activity and dietary behavior change: An updated systematic review. American Journal of Preventive Medicine, 42, 81-88.

Han, T. S., \& Lean, M. E. (1998). Self-reported waist circumference compared with the "Waist Watcher" tape-measure to identify individuals at increased health risk through intra-abdominal fat accumulation British Journal of Nutrition, 1, 81-88.

Jepson, R. G., Harris, F. M., Platt, S., \& Tannahill, C. (2010). The effectiveness of interventions to change six health behaviours: A review of reviews. BMC Public Health, 10, 538.

Kessler, R., \& Glasgow, R. E. (2011). A proposal to speed translation of healthcare research into practice: Dramatic change is needed. American Journal of Preventive Medicine, 40, 637-644.

Khoury, M. J., Gwinn, M., \& Ioannidis, J. P. A. (2010). The emergence of translational epidemiology: From scientific discovery to population health impact. American Journal of Epidemiology, 172, 517-524.

Lane, C., Huws-Thomas, M., Hood, K., Rollnick, S., Edwards, K., \& Robling, M. (2005). Measuring adaptations of motivational interviewing: the development and validation of the behavior change counselling index (BECCI). Patient Education and Counselling, 56, 166-173.

Larimer, M. E., Palmer, R. S., \& Marlatt, G. A. (1999). Relapse prevention. An overview ofcognitive-behavioral model. Alcohol Research \& Health, 23, 151-160.

Lindström, J., Ilanne-Parolla, P., Peltonen, M., Aunola, S., Eriksson, J. G., Hemiö, K., . . . Tuomilehto, J. (2006). Sustained reduction in the incidence of type 2 diabetes by lifestyle interventions: Follow-up of the Finnish Diabetes Prevention Study. The Lancet, 368, 1673-1679.

Milat, A. J., King, L., Baumam, A. E., \& Redman, S. (2012). The concept of scalability: Increasing the scale and potential adoption of health promotion interventions into policy and practice. Health Promotion International. Advance online publication. doi: 10.1093/heapro/dar097

Moroshko, I., Brennan, L., \& O’Brien, P. (2011). Predictors of dropout in weight loss interventions: A systematic review of the literature. Obesity Reviews, 12, 912-934.

Moyers, T. B., Martin, T., Manuel, J. K., Miller, W. R., \& Ernst, D. (2007). Revised Global Scales: Motivational interviewing treatment 
integrity 3.0 (MITI 3.0). Albuquerque: University of New Mexico, Centre on Alcoholism, Substance Abuse and Addictions.

National Health and Medical Research Council. (2003). Dietary guidelines for Australian adults. Canberra: Author.

O’Hara, B. J., Bauman, A. E., King, E., \& Phongsavan, P. (2011). Process evaluation of the advertising campaign for the NSW Get Healthy Information and Coaching Service ${ }^{\circledR}$. Health Promotion Journal of Australia, 22, 68-71.

O'Hara, B. J., Phongsavan, P., Venugopal, K., \& Bauman, A. E. (2011). Characteristics of participants in Australia's get healthy telephone-based lifestyle information and coaching service: Reaching disadvantaged communities and those most at need. Health Education Research, 26, 1097-1106.

Palmer, S., Tibbs, I., \& Whybrow, A. (2003). Health coaching to facilitate the promotion of healthy behaviour and achievement of health-related goals. International Journal of Health Promotion and Education, 41, 91-93.

Popkin, B. M., \& Gordon-Larsen, P. (2004). The nutrition transition: Worldwide obesity dynamics and their determinants. International Journal of Obesity, 28, 82-89.

Population Health Division. (2009). New South Wales Population Health Survey. Retrieved from http://www.health.nsw.gov.au/ publichealth/surveys/phs.asp
Prochaska, J. O., DiClemente, C. C., \& Norcoss, J. C. (1992). In search of how people change: Application to addictive behaviors. American Psychologist, 47, 1102-1114.

Rollnick, S., \& Miller, W. R. (1995). What is motivational interviewing? Behavioural and Cognitive Psychotherapy, 23, 325-334.

Rychetnik, L., Bauman, A., Laws, R., King, L., Rissel, C., Nutbeam, D., . . . Caterson, I. (2012). Translating research for evidence-based public health: Key concepts and future directions. Journal of Epidemiology \& Community Health. Advance online publication. doi:10.1136/jech-2011-200038

Rychetnik, L., Frommer, M., Hawe, P., \& Shiell, A. (2002). Criteria for evaluating evidence on public health interventions. Journal of Epidemiology \& Community Health, 56, 119-127.

Sallis, J., Grossman, R. M., Pinski, R. B., Patterson, T. L., \& Nader, P. R. (1987). The development of scales to measure social support for diet and exercise behaviors. Preventive Medicine, 16, 825-836.

Smith, B. J., Marshall, A. L., \& Huang, N. (2005). Screening for physical activity in family practice: Evaluation of two brief assessment tools. American Journal of Preventive Medicine, 29, 256-264.

Wright, J. L., \& Scott, J. A. (2000). The Fat and Fibre Barometer, a short food behaviour questionnaire: Reliability, relative validity and utility. Australian Journal of Nutrition and Dietetics, 57, 33-39. 